\title{
BREVE HISTORIA PERSONAL DE LA ANTROPOLOGÍA CHILENA
}

\section{A Brief Personal History of Chilean Anthropology}

\section{FRESIA MARÍA SALINAS*}

Fecha de recepción: 12 de mayo de 2021 - Fecha de aprobación: 2 de octubre de 2021

\section{Resumen:}

En este texto expongo, desde una perspectiva muy y explícitamente personal, algunas ideas sobre el despliegue de la antropología en Chile, en base a conversaciones con colegas, escuchas y experiencias vividas, lecturas de publicaciones diversas y un algo de reflexión, en dos momentos de mi (bastante marginal) incursión disciplinar.

Palabras clave: antropología en Chile, antropología chilena, avances y retrocesos, hitos, tendencias.

\section{Abstract:}

Within this text, I expose, from a very and explicitly personal perspective, some ideas about the deployment of anthropology in Chile. This is based on conversations with colleagues, hearings and lived experiences, readings of various publications and a bit of reflection, in two moments of my (very marginal) disciplinary foray.

Keywords: anthropology in Chile, Chilean anthropology, advances and retreats, milestones, trends. 


\section{Presentación}

En las páginas que siguen, aventuro algunas notas sobre mi visión de partes de la antropología en nuestro país, divididas en dos secciones, separadas entre sí por 18 años. La primera es una versión ligeramente actualizada de una presentación que me tocó realizar en 2003, a nombre del Colegio de Antropólogos de Chile, en el $51^{\circ}$ Congreso de Americanistas organizado en Santiago ${ }^{1}$. La segunda fue escrita en estos días y en ella relato -de nuevo, desde una mirada que no es ni pretende ser sino personal (por ende, parcial, sesgada y con puntos ciegos)- acerca de lo ocurrido en el devenir del ejercicio disciplinario durante el transcurso de este siglo.

\section{3 - ¿Antropología chilena? Su trayectoria como relato de vida}

Quisiera presentar a ustedes un par de apuntes sobre la situación de la antropología en Chile, conformados por retazos y recortes provenientes de diferentes fuentes: la experiencia ${ }^{2}$ personal de terreno, por así decir, que incluye, en mi caso, un terreno que se prolonga por unas cuantas décadas vividas; las conversaciones con miembros de la comunidad -entendida esta en una acepción más categorial que de sentido-; la observación flotante, que incluye las escuchas laterales; y documentos escritos de variado origen, tanto disciplinario como del medio social amplio.

\section{Primero: retazos y recortes}

Hace unos días, una joven antropóloga social escribió en el diario El Mercurio de Santiago sobre la necesidad de diálogo:

Qué acción más humana que conversar. Soy de aquellas personas dispuestas a sostener una conversación casi sobre cualquier cosa y con quien sea [...] Pero no sé qué nos pasa [...], que cada vez estamos conversando menos y peor [...] La clave, agrega, es que hemos perdido las confianzas básicas que nos permitían sentarnos a dialogar sin sentirnos amenazados. Me preocupa que como sociedad estemos encerrándonos cada vez más en pequeños compartimentos, en reductos que nos aseguran la homogeneidad necesaria para sentir que quien está al frente es un ser confiable. (Ivanovic, 2003, p. 15)

Pese a que podría considerarse a la antropología como una disciplina que, en parte importante, se desarrolla en la conversación -Rapport (1997) propone incluso una antropología de la conversación-, he escuchado a numerosos antropólogos que reclaman por la situación vivida entre nuestros propios pares, situación que conciben como de desunión y de desconocimiento mutuo -posiblemente deba expresarse al revés: desconocimiento y desunión-. El ex presidente del Colegio de Antropólogos, Juan Carlos Skewes, haciéndose eco de esta percepción, expresó en el lanzamiento de las Actas del IV Congreso Chileno de Antropología: "Nuestra comunidad está muy fragmentada".

En el mismo medio de comunicación recién mencionado, escribe un lector (en la sección "Cartas al Director", 2003) que el financiamiento de la actividad científica en Chile depende de los cada vez más escasos aportes de Fondecyt. 
Algunos colegas realizan investigaciones de largo plazo financiadas por este medio $-\mathrm{y}$, además, vía Fondart, lo que señala un tipo de orientación del trabajo antropológico-. Otros, logramos obtener, de vez en cuando, fondos extranjeros para realizar estudios de diversa índole, aunque mayormente dedicados a la superación de problemas sociales y culturales.

En tercer lugar, rechazos y apoyos reciben quienes, habiendo tenido un papel importante en el desarrollo de la disciplina en Chile, no han realizado estudios de pregrado en antropología sino en otras áreas. Rechazo, por esa misma razón, y apoyo, por su relevancia académica (también a nivel de políticas nacionales) y porque se asimila al historial de los maestros fundadores de la antropología mundial, que fueron abogados, filósofos, literatos, físicos, ingenieros... En tiempos más contemporáneos, algunos hitos, como Geertz y Clifford, también han llegado a la antropología desde otros ámbitos del conocimiento.

Segundo: un breve relato de vida

Hace unas semanas, fui invitada por un colega a hablar de antropología a algunos alumnos de la cátedra de Antropología en Chile, de la carrera de antropología de la Universidad Academia de Humanismo Cristiano. Mi estimado colega me señaló vagamente un tema que podía ser de interés para estos alumnos de quinto año: Stuchlik, su paso por Chile a comienzos de los años setenta, época en la cual yo estudiaba antropología y tuve la fortuna de ser su alumna. Siento, en parte, que me invitó porque, según me había expresado alguna vez, yo hacía "cosas entretenidas" y, en parte, por mi edad.
Abreviando la anécdota, me encontré con la sorpresa de que la cátedra era de José Bengoa, que era su primera clase, puesto que venía recién de hacer clases en Europa, que la persona con quien habíamos fijado la fecha era su ayudante y no solo no estaba en ese momento, sino que jamás le había avisado al profesor titular acerca de mi visita, por lo que este no tenía ninguna noticia al respecto. Encantada de liberarme de este compromiso, me apresuré a expresar mi conformidad de volverme para mi casa de inmediato. No obstante, y con gran presencia de ánimo y ya que estaba yo allí, el profesor Bengoa decidió que se concretase esta reunión. Así, me vi frente a un grupo de personas que me miraban con caras serias y que, como es costumbre en nuestras aulas, iban llegando de a poco, atrasados.

Ofrecí dos opciones de conversación: la primera, en referencia a la antropología de la cual había sido testigo; la segunda, en torno a la interrogante sobre la existencia de una antropología chilena. En fin, emprendí la primera y terminé con la segunda. Con la mayor amabilidad, Bengoa sintetizó mi intervención con el siguiente comentario: un relato aparentemente sencillo que, sin embargo, exponía la trayectoria de la antropología en Chile. Una síntesis de aquello expongo a continuación.

Se inicia esta trayectoria con la llegada de investigadores extranjeros al país, atraídos por la posibilidad de trabajar con los exóticos indígenas latinoamericanos; en nuestro caso, mayormente mapuches. Estudios como los de las primeras épocas de profesionalización se dedican a las sociedades o culturas -dependiendo del origen nacional de los antropólogos - en un aislamiento que después llegaría a parecer insólito e irreal. Stuchlik estudió durante 
varios años las estructuras organizativas de un grupo de comunidades indígenas en la zona de Cholchol, cercana a Temuco. Más adelante, las investigaciones se volvieron "interculturales" (mi tesis de grado trató de la aplicación de las leyes indígenas en juicios sobre tierras).

Llegaban a Chile, entonces, antropólogos de Estados Unidos, Inglaterra, Escocia, Noruega, Francia, para mencionar solo la procedencia de mis propios profesores, varios de ellos doctorandos que habían sabido del trabajo disciplinario que se llevaba a cabo en el sur del país. La vida académica, alimentada por los conocimientos que cada cual traía y que daba origen a intensos debates desarrollados en la ya lingua franca, el inglés, permitió, por una parte, un fecundo aislamiento intelectual $y$, por otra, una participación colateral en el movimiento ideológico nacional. La novedad de este socialismo ganado en las urnas también era un fuerte foco de atracción para especialistas extranjeros.

Con el golpe de Estado de 1973, los cambios fueron enormes para quienes estábamos estudiando $y$, evidentemente, para quienes trabajaban como profesionales. Se cerraron escuelas de antropología, nuestros profesores debieron partir, quedando los que no eran cuestionados a cargo de estos botes a medio hundir. En la escuela de la Universidad Católica en que me formé, de nuevo tuvimos la fortuna de contar con docentes como los Melville, Marjorie (Margarita) y Thomas, doctorandos norteamericanos. Nuestra especialización fue transformada en la que se consideró menos conflictiva: la etnolingüística. Rindo homenaje desde aquí a Adalberto Salas, erudito especialista en lenguas indígenas de Chile, recientemente fallecido, uno de los directores que en esos tiempos difíciles encabezó la Escuela. Unos años después del golpe, esta también corrió la misma suerte de la de la Universidad de Concepción y fue cerrada ${ }^{3}$.

Los años siguientes fueron los de las organizaciones no gubernamentales (ONG), las organizaciones al alero de la Iglesia ${ }^{4}$ y del trabajo para organismos estatales. De una $u$ otra forma, también los antropólogos debíamos subsistir, ojalá realizando aquello para lo cual nos habíamos formado. Fueron los años de la antropología aplicada. Esto no quiere decir que antes solo se trabajara en investigación pura; los antropólogos solían entregar sus conclusiones a las instituciones que parecieran tener un rol que jugar en los destinos de los grupos estudiados; que fuesen tomadas en cuenta 0 no, es otro asunto.

Hace un par de años, cuatro alumnas de la Universidad Bolivariana ${ }^{5}$ iniciaron un trabajo sobre la antropología en Chile durante la época de la dictadura. La denominaron la "época oscura", por la condición de semiclandestinidad del quehacer antropológico que, si bien permanecía como disciplina académica impartida en universidades, era mirado con sospecha por las autoridades de gobierno, y por los propios antropólogos, cuando sus pares trabajaban para ciertos organismos relacionados con el gobierno.

En esa etapa quisiera no pasar por alto el papel jugado por el Colegio de Antropólogos. Los miembros del Colegio tuvieron una destacada participación en la continuidad de la disciplina en Chile. Las sesiones, que se llevaban a cabo en Santiago, se expandían por el resto del país mediante modestas cartas y folletos -que hoy consideraríamos muy precarios, pero que eran bienvenida lectura para quienes vivíamos fuera de la capital-. Más todavía, a veces llegaban 
a constituirse en el solo lazo que todavía nos vinculaba con la profesión.

La llegada de la democracia coincidió, en mi caso particular, con la vuelta al trabajo. Habiendo sido despedida de una universidad a comienzos de los años ochenta -extraoficialmente, por "enseñar a pensar a los alumnos"-, inicié una nueva etapa profesional en Santiago, en una ONG, realizando estudios urbanos. Poco después, retomé la actividad docente, con unas horas de clases.

La investigación urbana en Chile se inició en los años cincuenta, con los estudios de Munizaga (1961), como todos sabemos. A comienzos de los ochenta, Marjorie Melville (1976) trabajó con mapuches migrantes en Santiago, como parte de su investigación en valores centrada, principalmente, en comunidades rurales de la IX Región. En los noventa, la actividad investigativa de la Escuela en la que trabajo $^{6}$ fue, casi en su totalidad, urbana: salud, juventud, pobreza, en barrios, comunas, campamentos citadinos, han sido temas y lugares que hemos tratado en evaluaciones de proyectos y programas para diferentes organismos. Sin embargo, los terrenos de los alumnos han mantenido la locación ajena a su propia situación urbana de vida, y se han realizado en Ralco; en Temuco y pueblos aledaños; en distintos puntos de la Carretera de la Fruta, y en la costa central, entre otros. Pese al cambio de ubicación, la antropología todavía parece llamada a desarrollarse en la ajenidad; parte de su fascinación depende de ello.

En la urbe, la complejidad y la influencia de la globalización parecen destacarse más que en los cada vez más reducidos lugares rurales, que también fueron tema y marco de estudio disci- plinario, sobre todo en los setenta y ochenta. Posiblemente, mientras más escasos se hagan, más antropólogos recibirán, quiéranlo o no: serán los espacios de exotismo, todavía la miel para los paladares antropológicos. En la ciudad, los exóticos son, hoy, los pobres, los habitantes de campamentos; los marginales al límite (travestis, trabajadoras y trabajadores sexuales, los sin casa habitantes de las calles); los jóvenes agrupados, en pandillas, en bandas; las llamadas sectas o las minorías sexuales.

Todavía son estudiados los indígenas urbanos, algunos de los cuales ya están prohibiendo la entrada a sus reuniones a periodistas y antropólogos. Esto puede considerarse de tres maneras, al menos. En primer lugar, estaríamos alcanzando el nivel de reconocimiento popular de los periodistas, es decir, dejamos de ser unos desconocidos, al menos para quienes más han tenido que soportarnos. Un tema de preocupación mundial entre los antropólogos -incluso en Gran Bretaña, cuna de la antropología social moderna- ha sido la popularización de la disciplina, en el sentido de señalarse la necesidad de que el público en general la conozca; para ello, se ha trabajado con la prensa.

En segundo lugar, sería interesante indagar en este fenómeno: ¿cuáles son las razones específicas esgrimidas por quienes rechazan la participación antropológica? ¿Cuestiones culturales, experiencias concretas vividas, antipatías personales? Y quienes todavía aceptan nuestras intromisiones, en fin, ¿por qué lo hacen?

En tercer lugar, pese a lo anterior, la antropología como disciplina parece seguir siendo atractiva, puesto que cada vez más, en distintos lugares de la Tierra, se la estudia y se 
llevan a cabo investigaciones antropológicas. Esto significa que quienes alguna vez fueron "objeto de estudio" o partícipes del "objeto" han visto ciertas ventajas en ser ellos mismos antropólogos. En África, en Asia, en América Latina, en Chile, tenemos crecientes cantidades de estudiantes y profesionales antropólogos de origen indígena. Quienes alguna vez pensamos ser la voz de los sin voz, estamos siendo reemplazados por las voces de los que están alzándola. Y, paradojalmente quizás, por la mediación de nosotros mismos.

Una mirada a la antropología en Chile resulta ser, como se ve, una revisión desde la experiencia vivida y una muestra de la antropología mundial y su trayectoria. Lejanos, nosotros, mantenemos nuestras conexiones con el desarrollo de la disciplina. Somos parte, así, de la comunidad planetaria.

\section{Tercero: ¿una antropología chilena?}

Puestas las cosas de esta manera, ¿existe algo que podamos denominar, propiamente "antropología chilena"? De otro modo, ¿puede esta existir? Si se diera el caso de que proclamásemos su existencia posible, ¿cuáles serían sus características? No pretendo una respuesta fácil del tipo de "la antropología chilena es todo aquello que hacemos las y los antropólogos chilenos", a semejanza de la definición de etnografía de Monaghan y Just: "etnografía es lo que hacemos los antropólogos" (2000, p. 13). O una respuesta al estilo de Moore (1999): la historia de la antropología es la antropología. Tampoco quisiera limitarme a lo recién planteado respecto de nuestra inserción en la comunidad antropológica mundial.
Varios elementos podrían tenerse en cuenta en un intento de dar respuesta a estas interrogantes. En primer lugar, se puede echar mano al trabajo de los antropólogos chilenos en el contexto contemporáneo. Aunque reconocemos la amplitud de los ámbitos laborales en los cuales hoy encontramos a antropólogos, asimismo debemos reconocer que estamos, todavía, en situación de abrir espacios. Esto se relaciona, en nuestra perspectiva, con elementos tales como las percepciones que en el país existen respecto de la disciplina, sobre todo más allá de lo académico. Añado a ello la preocupación por la popularización de la disciplina, tema de interés en diferentes partes del mundo.

¿Qué constituye a una antropología chilena? ¿El estudio de los pueblos, etnias, culturas o sociedades indígenas originarias? Desde fuera de nuestros límites geográficos y académicos, parece de gran interés todo aquello referente a estos pueblos; son los exóticos de Chile, del sur andino. La tradición antropológica mundial avalaría este objeto como central para una antropología chilena, latinoamericana, indoamericana, americanista. De la misma manera, el área geográfica sería un elemento diferenciador de otras antropologías, aunque los mapas del territorio se traslapasen. No obstante, se me ha señalado que un área antropológica chilena es o irrelevante o inexistente: de lo que se trata es de una antropología latinoamericana o americana, como lo demuestra el $51^{\circ}$ Congreso de Americanistas en el que se comparte esta reflexión.

¿Será el tratamiento de ciertos temas lo que, además, caracterizaría a esta antropología chilena? Por ejemplo, los temas de derechos humanos, de las violaciones a estos derechos, de los detenidos desaparecidos, aparecieron y 
adquirieron relevancia y presencia pública en la etapa posdictadura ${ }^{7}$. $Y$ vinculado con este tema, ¿podríamos mencionar una ética de la antropología chilena que la distinga de las demás? Cuando trabajamos para organismos que apoyan el despojo de tierras y aguas de los pueblos originarios, o cuando trabajamos apoyando a los más desposeídos de nuestro país, ¿hay consideraciones éticas que nos validen, en cada circunstancia?

En fin, si, como Herzfeld (1997) consideramos que la antropología es la práctica de una teoría, o se construye en la relación de datos empíricos y teorías, ¿podemos decir que hemos generado teoría(s) propia(s) y distintiva(s)? En distintos momentos de reunión de antropólogos chilenos, se ha podido escuchar el reclamo por la falta de teorización. Aparentemente, nos dedicamos sobre todo a la práctica, o a la producción de información etnográfica, y no al proceso fundamental de creación teórica -nuestras orientaciones de sentido, entonces, nos serían prestadas-.

Nuestro aporte a la antropología mundial o global consistiría, entonces, en etnografía de las etnias indígenas que habitan este pequeño rincón del planeta (como también son habitantes de Perú, Bolivia y Argentina).

Evidentemente, la discusión de la existencia o no de una antropología chilena pasa por la tradicional distinción entre una antropología como ciencia -universalista- y una antropología de orientación humanista, distinción que parece estar siendo, otra vez, punto de debate. Si concebimos a la chilena como parte de la antropología cientificista, pues el problema de su existencia o no se diluye: solo interesa, desde allí, que forme parte de la corriente global de pensamiento, de producción de conocimiento acumulativo y de procedimientos validados por la comunidad científica. Pero surge el problema de nuestro aporte a esta comunidad de saber.

Por otra parte, insisto en preguntar si es posible otra cosa, que no sea esa pertenencia a una comunidad disciplinaria y que nos deja la posibilidad de copiar. Esto quiere decir, en la práctica, que aplicamos la producción teórica creada por otros; significa, por ejemplo, adaptar teoría a nuestra realidad. Nada de esto es irrelevante en el concierto del desarrollo de la ciencia antropológica. Quisiera, empero, ir más allá.

Como herencias de una posmodernidad que se dice se bate en retirada ya desde 1998 en la antropología, nos han quedado un énfasis en la reflexividad y la importancia de lo local. Utilizando terminología que también va quedando obsoleta, quisiera recordar los aportes de dos distinguidos antropólogos que escriben desde sectores periféricos del mundo antropológico: García Canclini (1989), con el concepto de culturas híbridas, y Karim (1996), con el de antropología popular. Aquí, justamente, reside el interés prospectivo de una mirada -incluso una tan superficial como esta- a la antropología chilena.

Creo que nuestro papel a futuro debiera estar en la creación y la producción de pensamiento antropológico que traspase límites, pero a partir del reconocimiento de esas mismas fronteras; del reconocimiento de nuestra situación periférica, tercermundista; de nuestras prácticas, académicas pero también externas a la academia. Del reconocimiento y la aceptación de quienes somos: integrados al mundo, pero también isleños; viajeros reales y virtuales, pero también profundamente localizados y territoria- 
lizados. De la conciencia de nuestros sujetos, temas, área, ética e, incluso, de la imaginación teórica que sea capaz de unir todo esto y lo ofrezca al mundo. Esto, por último, no significa rechazar aquello que viene de fuera, en pro de un bizarro nacionalismo antropológico, sino sacar partido a nuestras peculiares circunstancias; significa traer lo mejor del mundo y entregarle lo mejor de nosotros.

\section{Cuarto: entretejiendo recortes y retazos}

Catalina Ivanovic, la antropóloga social con cuyas palabras iniciara esta presentación, termina su columna con la propuesta de llevar a cabo "una conversación verdadera, en que [cada cual] se haga presente con lo suyo. Así caerá el miedo y descubriremos a ese otro" (2003).

Recojo sus dichos, no solo por lo que ellos valen sino porque la inclusión de una columna escrita por una antropóloga en un diario no es un hecho habitual. Además, porque lo escribe una mujer, lo que refleja la feminización de la antropología, fenómeno que ya ha sido destacado dentro de la comunidad antropológica mundial. Igualmente, porque se trata de una antropóloga social de 25 años, es decir, que está iniciando su quehacer profesional.

Si el anterior presidente del Colegio hacía notar el problema de la ausencia de un colectivo, el actual ha señalado que este es un ente simbólico, con poder simbólico, o sea, con la capacidad de congregar y proyectar. Si somos capaces de hacernos cargo, como personas individuales y como miembros de una organización colegiada, de nuestras responsabilidades -derivadas incluso de las características de nuestra profesión-, es decir, si abrimos nuestra mirada a los enfoques científico y humanista, a los intereses académicos, políticos y éticos; a los aportes de quienes, procedentes de otras formaciones disciplinares, han llegado a ser relevantes en la antropología; a los aportes de cada uno de nosotros, en forma integrada y no en términos de contradicciones excluyentes, creo que se incrementarán exponencialmente nuestras posibilidades de conocimiento y comprensión de la compleja realidad contemporánea.

\section{1 - Addendum: huellas de los días}

Han pasado casi veinte años desde que la primera parte de este texto fuera escrita y presentada. Como es de suponer, mucho ha ocurrido desde entonces. De todo ello, y dado que mi propósito es dar una cuenta muy breve y personal de este devenir de la disciplina en nuestro país, sumo en esta segunda parte un poco de esto y un poco de aquello, de lo que recuerdo, de lo que he leído y de lo que me han contado, de lo que han sido -de nuevo, insisto, en mi mirada, parcial, sesgada, con puntos ciegos- hitos, retrocesos y avances.

Si a principios de este siglo escribí que esta antropología reflejaba quiénes somos, como profesionales/académicos, miembros de este país y de sus circunstancias, y, a la vez, participantes periféricos de la antropología mundial, hoy puedo volver a señalar algo similar. Al mismo tiempo, como es obvio, no todo sigue igual. Mucho se mantiene, entremezclado con cambios significativos.

En primer término, la historia de la antropología en este sur del mundo ha despertado entre nosotros y nuestros amigos la atención y el interés por desplegarla, desde el siglo pasado 
(por ejemplo, Garbusky, 1998, sobre la antropología en la Universidad de Concepción antes de la dictadura). Orientados por diversas perspectivas, han ido apareciendo estos relatos, objetivados y también subjetivos, del paso y el desenvolvimiento de la antropología en Chile (otros ejemplos: Morales y Cancino, 2003, sobre antropología en el sur de Chile; Castro, 2014, que revisa 60 años de antropología en el país); enlistados, van sumando alrededor de 100 publicaciones $^{8}$. La pregunta que nos hacíamos por la existencia de una "antropología chilena" va quedando así obsoleta; si tanto se narra acerca de ella, será, plausiblemente, porque de una u otra forma se reconoce su existencia (o su presencia como una entidad distinguible de otras de su tipo). Con esto, compartimos el creciente interés de colegas de otros países latinoamericanos que han venido escribiendo sobre sus propias antropologías nacionales (una muestra relevante es el artículo de la argentina Rosana Guber en la revista del Royal Anthropological Institute (RAI), Anthropology Today, publicado en 2002).

En segundo lugar, y a propósito de orientaciones o perspectivas, no puedo dejar de mencionar lo que considero un momento cumbre de la antropología en Chile, que apareció y fue siendo desarrollado en años en que alumnas y alumnos de la Universidad Bolivariana ${ }^{9}$ preguntaban si había o no algo que pudiera denominarse, con fundamento, "antropología chilena".

La carrera de antropología de la Pontificia Universidad Católica, sede Temuco, cuyos inicios estuvieron en un programa liderado por el doctor Milan Stuchlik a principios de la década de 1970, continuó con altos y bajos, con cierres y reaperturas hasta que, volviendo de estudiar en Belfast, la doctora Teresa Durán -alumna de la primera generación- fundara el Centro de Estudios Socioculturales (CES) y se hiciera cargo de ella. Junto con un equipo de antropólogas y antropólogos, principalmente formados en Temuco, Teresa ${ }^{10}$ desarrolló lo que se denominó la "antropología interactiva", fecundo enfoque que aunó teoría y práctica de la disciplina en cuanto dominio formal del conocimiento con las circunstancias del tiempo-espacio en que estaba siendo ejercitada, y con una visión ética de compromiso explícito con las personas con las que se colaboraba - compartiendo, asimismo, autorías-.

Tiempo, circunstancia, lugar, personas/ comunidades, esto es, para usar otra terminología que estuvo en boga hace años atrás, una antropología a la vez cosmopolita y local. Una cantidad de publicaciones dieron fe de este enfoque, que entrelazaba estrechamente todos esos elementos constitutivos. La cumbre a que me refiero ${ }^{11}$ se reflejó en un número de la revista española Anthropos dedicado a textos de miembros de ese equipo. La doctora Durán escribió allí "Duplicando la antropología en la Araucanía de Chile" (2005), título que no dice todo lo que el artículo contiene, ni lo más importante: esa "duplicación" fue un avance disciplinario que bien vale la pena considerar una y otra vez.

Entre los avances, igualmente contaría la apertura de carreras de antropología de pregrado y, sobre todo, de posgrados de magíster y doctorado en diferentes universidades, entre ellas, la Universidad de Los Lagos, la Universidad Alberto Hurtado, la Universidad Academia de Humanismo Cristiano, la Universidad Católica del Norte, la Universidad de Tarapacá y la Pontificia Universidad Católica de Chile. Evidentemente, tales aperturas manifiestan, por un 
lado, el interés que los estudios antropológicos académicos y profesionales siguen despertando en nuestro país y, por otro, el nivel de madurez que la antropología ha ido alcanzando. Ya no es, como lo fue, indispensable salir al extranjero para obtener el grado académico superior (junto con docentes nacionales, profesoras y profesores extranjeros siguen colaborando en estos programas doctorales).

Y, al revés, quizás entre los retrocesos está el cierre de carreras de antropología, como ha sido el caso de la Universidad Bolivariana y de la Universidad ARCIS. La primera, aunque ya no imparte antropología, al menos sigue existiendo como institución; la segunda ha desaparecido. Víctimas de diversas circunstancias: me atrevería a mencionar, entre ellas, la fatiga de material, esto es, la pérdida del entusiasmo inicial debido al cambio de dueños, de planes y orientaciones y a la consecuente pérdida del equipo en formación, así como la creciente competencia. La imperante cultura de la auditoría, por otra parte, no fue siempre tenida en cuenta, lo que terminó acarreando problemas académicos, económicos y administrativos. Desde un punto de vista antropológico, ¡un error en la visión culturalista proyectiva!

Por último, y saltándome tantos años, escuelas, maestras y maestros... termino con dos menciones, igualmente breves y que veo intercaladas o íntimamente entrelazadas. Una, relativa a los temas de interés, la otra, relacionada con la orientación antropológica que da fundamento a cualquier planeamiento que se diga antropológico (o etnográfico dentro de la disciplina).

En estos últimos años tan llenos de acontecimientos no solo nacionales sino globales, dos han sido los que han concitado el mayor interés a nivel de los medios de comunicación, colectivos y personas individuales: el denominado estallido social y la pandemia de COVID-19. Con cada vez menor acceso a la cotidianeidad fuera de casa, y por opción poco aficionada a la televisión, incluso como ermitaña o con la cabeza enterrada en la arena, difícilmente habría podido quedar ajena a estos acontecimientos. Tampoco ajenos quedaron las y los antropólogos en ejercicio. Entre las publicaciones a las que he tenido acceso -actualmente, muchas online- menciono a Márquez (2020) y los escritos (y audiovisuales) de antropólogas y antropólogos y estudiantes de la Universidad Alberto Hurtado, que entre sus prácticas etnográficas difundieron variadas experiencias durante el estallido de 2019 (VV.AA., 2019). Y sobre la pandemia, me permito señalar los dos volúmenes de crónicas del COVID-19, con textos de varios autores, editadas por Mauricio Osorio $(2020,2021)$ y publicadas en Coyhaique.

¿Por qué hago referencia a esas producciones? La respuesta va de la mano con sus orientaciones de base. Se trata de etnografías personales, de autoetnografías. Como sabemos, la autoetnografía ha sido tendencia creciente en la antropología de este siglo. Descendiente del Malinowski autor de su Diario en el estricto sentido del término (1989 [1967]), de sus nietos los posmodernos de fines del siglo pasado (Behar, 1996; Crapanzano, 1980; Rabinow, 1992; y también Barley, 1986), con otro nombre presentada en Chile en las cuidadas publicaciones de antropólogos del Museo Chileno de Arte Precolombino, en las Etnografías mínimas que publicara Daniel Quiroz (2007) y en la obra de Juan Carlos Olivares en ese volumen (2007). Y así contando ${ }^{12}$. Una etnografía, unos escritos que terminan por convertirse en poemas (sobre el COVID, Pablo 
Méndez, 2007), en volúmenes de poesía (Ivonne Valenzuela y Juan Pablo Loo, 2008, en Gracias por el favor concedido, y especialmente Ivonne Valenzuela, 2019, en Bioversidad; y Cristóbal Palma con su Lumpenpoesía, 2020).

Con esto, entiéndase que no estoy descartando los textos contemporáneos de antropólogas y antropólogos chilenos que escriben sobre estos y otros temas-entre los cuales están la antropología y las tecnologías de la información y la comunicación (TIC) ${ }^{13}$, el(los) mundo(s) digital(es), las neurociencias, etc., etc.- y cuyas orientaciones son otras; en absoluto. Cada uno tiene su propio mérito. En forma especial recuerdo aquí los numerosos trabajos desarrollados por colegas del sur de Chile, muchos de los cuales no traspasan los límites de informes, importantes en distintas instancias de acción, pero que permanecen sin difusión fuera de esas a veces bastante estrechas fronteras.

\section{Notas}

${ }^{1}$ La presentación sintetizaba el texto, cuya versión escrita completa estuvo alguna vez disponible en la página web del Colegio.

${ }^{2}$ Entiendo "experiencia" aquí en el sentido en que lo plantea Throop (2003).

${ }^{3}$ Alejandro Herrera, Ivonne Jelvez y Roberto Morales, distinguidos colegas y ex alumnos de antropología de la Universidad Católica de Temuco, recuerdan esos años en que vivimos en peligro durante una reciente conversación con Héctor Mora en el marco de las XII Jornadas de Antropología y Arqueología (Mora, 2021). Mencionaban, en tal ocasión, que "muchos temas no podían ser tocados", y especialmente estaba excluido lo que se relacionara con teoría crítica. Asimismo, "todo lo que hacíamos", "era clandestino"; para ir a terreno "había que pedir permiso a la Gobernación". Pero, a la vez, se generaron relaciones estrechas y en general de confianza entre las y los compañeros de carrera, al contrario de lo que estaba ocurriendo en el contexto nacional.

${ }^{4}$ Entre las ongs independientes, destaco el Centro Asesor y Planificador de Investigación y Desarrollo (CAPIDE), fundado por antropólogas y antropólogos formados en la P. Universidad Católica sede Temuco; $y$ entre las organizaciones al alero de Iglesia, el Centro
Puesto que lo que aquí presento es una historia personal, me atrevo a destacar lo que subrayo, en la convicción de que no está (o no debería estar) fuera del canon tradicional de una antropología que se quiere científica, por cuanto cada quien que investiga, escribe, difunde, lo hace desde sí. $Y$ esa o ese que somos cada una, cada uno, como el principal instrumento del proceso investigativo que lleva a cabo, tiene el deber -y el derecho- de manifestar de manera explícita su punto de vista, su perspectiva, sus percepciones, sus emociones ${ }^{14}$ y sentimientos ${ }^{15}$. Su enfoque, ya sea que presente tablas estadísticas o versos. $\mathrm{O}$, tal vez, tablas y versos.

En fin, pues, seguimos (siguen, puesto que escribo desde el retiro) en este camino de re-crear y crear una disciplina, una profesión, que nunca, en ningún momento, ha dejado de apasionarme.

Ecuménico Diego de Medellín, en el cual desarrollaron parte de su relevante trabajo disciplinar la doctora Sonia Montecino y el doctor Rolf Foerster.

${ }^{5}$ Esas alumnas, cuyos nombres no mencioné en el texto original, fueron Isabel Arratia, Isabel Contador y Gricel González. Lamentablemente, he olvidado quién era la cuarta, pido disculpas por ello.

${ }^{6}$ Escuela de Antropología de la Universidad Bolivariana.

${ }^{7}$ En este caso, habríamos estado poniendo en práctica lo que Geertz (1996) destaca como labor disciplinar: ir tras los hechos.

${ }^{8}$ Esta cantidad me ha sido señalada recientemente por Leonardo Piña y Claudio Espinoza, en comunicación personal.

${ }^{9}$ Me refiero a estas y estos alumnos dado que trabajé en docencia e investigación en la carrera de antropología de esa universidad (1993 - 2009).

${ }^{10}$ Cabe destacar que la doctora Durán era profesora y trabajadora social; estas, sus dos primeras profesiones, marcadoras de su historia de vida, tuvieron también incidencia -como es de suponer- en su planteamiento general de la antropología y en el enfoque teóricopráctico específico que llevó a cabo y difundió en publicaciones y 
presentaciones en distintas partes del mundo.

${ }^{11}$ Esta cumbre fue más allá de la antropología de la Universidad Católica de Temuco; abarcó a la antropología ejercida en Chile, concebida, a mi parecer, como una antropología chilena propiamente tal.

12 Este 2021, en contexto de pandemia y confinamientos, en la Universidad de Santiago se ofreció un taller de investigación denominado Autoetnografía: el yo, la reflexividad y formas de vida, a cargo del antropólogo Dr. Cristián Rozas.

${ }^{13}$ Destaco el trabajo pionero del Dr. Francisco Osorio, sobre uso de programas computacionales para el manejo de data y sobre medios de comunicación masiva.

\section{Referencias bibliográficas}

Adams, S. \& Rice-Lively, M. (2009). Personality and research styles: Why we do the things we do. Journal of Education for Library and Information Science, 50(1), 50-58. Recuperado de thefreelibrary. com.

Barley, N. (1986). The innocent anthropologist: Notes from the mud hut. Londres: Penguin Books.

Behar, R. (1996). The vulnerable observer: Anthropology that breaks your heart. Boston: Beacon Press.

Breuer, F. (2004). Lo subjetivo del conocimiento socio-científico y su reflexión: Ventanas epistemológicas y traducciones metodológicas. Forum: Qualitative Social Research, 4(2). Recuperado de http:// nbn-resolving.de/urn:nbn:de:0114-fqs0302258.

Castro, M. (2014). A sesenta años de la antropología en Chile. Antropologías del Sur, 1(1), 43-64. Recuperado de http://revistas. academia.cl/index.php/rantros/article/view/770/878.

Crapanzano, V. (1980). Tuhami: Portrait of a Moroccan. Chicago: The University of Chicago Press.

Durán, T. (2005). Duplicando la antropología en la Araucanía de Chile. Anthropos, 207 (Antropología y sociedad. Una relación compleja, crítica y problemática. Propuesta de una antropología interactiva), 23-42

Friesen, L. (2021). Theories generate emotions. Academia Letters, Artículo 2682. Recuperado de http://doi.org/10.20935/AL2682.

Garbusky, E. (1998). La antropología en la Universidad de Concepción (1967-1973). Apuntes de un participante. En Colegio de Antropólogos de Chile, Actas del Tercer Congreso Chileno de Antropología (Tomo I, pp. 200-210). Temuco: Colegio de Antropólogos de Chile.

García Canclini, N. (1989). Culturas híbridas: Estrategias para entrar y salir de la modernidad. México: Grijalbo.

Geertz, C. (1996). Tras los hechos: Dos países, cuatro décadas y un antropólogo. Barcelona: Paidós.

González, A. (2017). In search of a sociological explanation for the emotional turn. Sociologia. Problemas e Práticas, 85, 27-45. Recuperado de https://journals.openedition.org/spp/3248.

\begin{abstract}
${ }^{14}$ ¿Dejo de lado la persona que soy cuando voy al campo, cuando escribo los resultados? Teniendo en cuenta el público destinatario, nuestros productos varían, pero en el escrutinio disciplinario, las razones o convicciones ónticas, epistemológicas y teóricas de quien investiga deben quedar claras.

${ }^{15}$ Por otra parte, como señala Friesen (2021), las teorías concitan sentimientos y los métodos se asocian con características de personalidad (Adams \& Rice-Lively, 2009). Igualmente, ver Breuer sobre lo subjetivo del conocimiento en ciencias sociales (2003) y González sobre el emotional turn (2017).
\end{abstract}

Guber, R. (2002). Antropología social: An Argentine diaspora between revolution and nostalgia. Anthropology Today, 18(4), 8-13.

Herzfeld, M. (1997) La antropología: Práctica de una teoría. Revista Internacional de Ciencias Sociales, 153. Recuperado de http://www. unesco.org/issj/rics153/herzfeldspa.html\#mhart.

Ivanovic, C. (2003). La necesidad de diálogo. El Mercurio, 13 de julio, Cuerpo E, 15.

Karim, W. (1996). Anthropology without tears: How a 'local' sees the 'local' and the 'global'. En Moore, H. (Ed.), The future of anthropological knowledge (pp. 115-138). Londres: Routledge.

Malinowski, B. (1989 [1967]). A diary in the strict sense of the term. Palo Alto: Stanford University Press.

Márquez, F. (2020). Por una antropología de los escombros: El estallido social en Plaza Dignidad, Santiago de Chile. Revista 180, 45, 1-13. Recuperado de http://www.revista180.udp.cl/index.php/ revista180/article/view/717/438.

Melville, M. (1976). The Mapuche of Chile. Their values and changing culture. Michigan: Ann Arbor.

Méndez, P. (2020). Décimas de la cuarentena. En Osorio, M. (Ed.), Crónicas de no estar en los otros: Miradas antropológicas a la crisis del COVID-19 en Chile y el mundo (Vol. 2, p. 9). Coyhaique: Nire Negro.

Monaghan, J. \& Just, P. (2000). Social \& cultural anthropology: A very short introduction. Oxford: Oxford University Press.

Moore, H. (1999). Anthropological theory at the turn of the century. En Moore, H. (Ed.), Anthropological theory today (pp. 1-23). Cambridge: Polity Press.

Mora, H. (Coord.-Mod.) (2021). Mesa redonda. Primeros titulados de antropología en la Universidad Católica de Chile, sede Temuco. Abriendo caminos al quehacer antropológico en el sur de Chile. Invitados: Ivonne Jelves, Roberto Morales, Alejandro Herrera. XII Jornadas de Antropología y Arqueología, Universidad Católica de Temuco, 18 de octubre. Recuperado de https://www.facebook.com/ carrera.antropolgia.1/videos/30132976814058/.

Morales, R. \& Cancino, R. (2003). La antropología desbordada: 
Las marcas y huellas del hacer antropología en territorio mapuche. En Richard, N. (Ed.), Movimientos de campo en torno a cuatro fronteras de la antropología en Chile (pp. 91-107). Guatemala: Instituto Centroamericano de Prospectivae Investigación. Recuperado de http://es.scribid.com.

Munizaga, C. (1961). Estructuras transicionales en la migración de los araucanos de hoy a la ciudad de Santiago de Chile. Santiago: Universitaria.

Olivares, J. C. (2007). Shamanes en la garúa: Antropología poética del Jesús Nazareno de Isla Caghach, archipiélago de Chiloé. En Quiroz, D. (Ed.), Etnografías mínimas (pp. 25-32). Santiago: Andros.

Osorio, M. (Ed.) (2020). Crónicas de no estar en los otros: Miradas antropológicas a la crisis del COVID-19 en Chile (Vol. 1). Coyhaique: Ñire Negro.

(Ed.) (2021). Crónicas de no estar en los otros: Miradas antropológicas a la crisis del COVID-19 en Chile y el mundo (Vol. 2). Coyhaique, Ñire Negro.

Palma, C. (2020). Lumpen poesía y otros poemas. Santiago: Camino.
Quiroz, D. (Ed.) (2007). Etnografías mínimas. Santiago: Andros.

Rabinow, P. (1992). Reflexiones sobre un trabajo de campo en Marruecos. Barcelona: Júcar.

Rapport, N. (1997). Edifying anthropology: Culture as conversation; representation as conversation. En James, A., Hockey, J. \& Dawson, A. (Eds.), After writing culture: Epistemology and praxis in contemporary anthropology (pp. 177-193). Londres: Routledge.

Throop, C. (2003). Articulating experience. Anthropological Theory, 3(2), 219-241.

Valenzuela, I. (2019). Bioversidad: Una larga visita al desierto. Santiago: Ceibo.

Valenzuela, I. \& Loo, J. P. (2008). Gracias por el favor concedido: Las animitas de Evaristo Montt, Elvira Guillén y Juana Guajardo. Antofagasta: Ercilla.

VV.AA. (2019). Microetnografías de la revuelta. Carrera de Antropología, Universidad Alberto Hurtado. Recuperado de https:// etnografíasdelarevuelta.blogspot.com/. 